\title{
ON SUMS OF LARGE SETS OF INTEGERS $\left({ }^{1}\right)$
}

\section{BY}

J. H. B. KEMPERMAN

1. Let $n$ be a fixed positive integer. Consider $k \geqq 3$ sets $A_{1}, \cdots, A_{k}$ of nonnegative integers, each containing zero, such that

$$
n \notin C \text {, }
$$

where

$$
C=A_{1}+\cdots+A_{k}
$$

consists of all the integers which can be written as a sum $a_{1}+a_{2}+\cdots+a_{k}$ with $a_{1} \in A_{1}, \cdots, a_{k} \in A_{k}$. If $S$ is a set of integers, we write

$$
S(x, y)=\sum_{x<s \leqq y ; s \in S} 1 \text {. }
$$

It is well known that (1.1) implies

$$
\sum_{i=1}^{k} A_{i}(0, n) \leqq k \frac{n-1}{2}
$$

Moreover, (see §3), if the equality sign holds in (1.2) then necessarily

$$
C(0, n)=\frac{n-1}{2}
$$

(hence, $n$ must be odd). Thus, (1.2) can be improved if in addition to (1.1) we require that

$$
\{0,1,2, \cdots, n-1\} \subset C,
$$

that is, $n$ is precisely the smallest positive integer not in $C$. Let

$$
\phi_{k}(n)=\min \left(k \frac{n-1}{2}-\sum_{i=1}^{k} A_{i}(0, n)\right),
$$

where $\left(A_{1}, \cdots, A_{k}\right)$ ranges over the $k$-tuples satisfying (1.1) and (1.3). It was shown by Erdös and Scherk [1] that (for $k \geqq 3$ )

$$
\alpha_{k} n^{(k-1) / k} \leqq \phi_{k}(n) \leqq \beta_{k} n^{(k-1) / k},
$$

where

$$
\alpha_{k}=\left\{2^{k / 2+4}(k-1) !\right\}^{-1}, \quad \beta_{k}=(k+1) 2^{2 k-3} .
$$

Received by the editors, January 5, 1963.

(1) This work was supported by the National Science Foundation, grant NSF-G-24470. 
This result will be sharpened to:

THEOREM 1.1. One has, for each $k \geqq 3$, that

$$
\lim _{n \rightarrow \infty} \phi_{k}(n) n^{-(k-1) / k}=k 2^{-(k-1) / k} \text {. }
$$

Note that $\phi_{2}(n)=0$; for, take $A_{1}=\{0,1, \cdots, m\}$ and $A_{2}=\{0,1, \cdots, n-1-m\}$; further on, we shall always assume that $k \geqq 3$. As will be seen, the result (1.6) can be generalized to other conditions of the type (1.3). One of the important tools is Lemma 3.2. Its proof is given in $\$ 5$ and is based upon an isoperimetric inequality which seems to be of independent interest. In $\S 3$, using Lemma 3.2, we easily obtain an inequality of the type (1.5) with $\beta_{k}=2 \alpha_{k}$. The proof of the stronger result (1.6) is given in $\$ 4$.

2. Let us first establish an upper bound on $\phi_{k}(n)$, namely,

$$
\phi_{k}(n)<k\left(1+\left(\frac{n}{2}\right)^{1 / k}\right)^{k-1} \text {. }
$$

By $\phi_{k}(1)=0$, we may assume $n \geqq 2$. Put

$$
p=1+\left[\left[\frac{n}{2}\right]^{1 / k}\right]
$$

thus, $p$ is the smallest positive integer satisfying

Letting

$$
\left[\frac{n}{2}\right] \leqq p^{k}-1
$$

$$
D_{i}=\left\{0, p^{i-1}, 2 p^{i-1}, \cdots,(p-1) p^{i-1}\right\} \quad(i=1, \cdots, k),
$$

each integer $0 \leqq y \leqq p^{k}-1$ has a unique representation as a sum $y=d_{1}+\cdots+d_{k}$ with $d_{i} \in D_{i}$. In particular,

$$
\left\{0,1, \cdots,\left[\frac{n}{2}\right]\right\} \subset D_{1}+D_{2}+\cdots+D_{k} .
$$

If $n$ is small it may happen that $n \in D_{1}+\cdots+D_{k}$, that is,

$$
n=\sum_{i=1}^{j} \varepsilon_{i} p^{i-1}
$$

where $1 \leqq j \leqq k, \varepsilon_{i} p^{i-1} \in D_{i}, \varepsilon_{j} \geqq 1$. But then

$$
n \leqq \varepsilon_{j} p^{j-1}+(p-1) \sum_{i=1}^{j-1} p^{i-1}<2 \varepsilon_{j} p^{j-1} .
$$

Now, modify $D_{j}$ by canceling the single element $\varepsilon_{j} p^{j-1}>n / 2$ (the other $D_{i}$ remain unchanged). This does not effect property (2.2). Moreover, we now have that 


$$
n \notin D_{1}+\cdots+D_{k} \text {. }
$$

Finally, we form $A_{i}(i=1, \cdots, k)$ as the union of $D_{i}$ and the collection of all integers $n / 2<x<n$ such that $n-x \notin E_{i}$, where

$$
E_{i}=D_{1}+\cdots+D_{i-1}+D_{i+1}+\cdots+D_{k}
$$

Clearly, the resulting $k$-tuple satisfies (1.1). It also satisfies (1.3). By (2.2), it suffices to show that $x \in A_{i}$ for some $i$, where $x$ is a given integer satisfying $n / 2<x<n$. If not, then the positive integer

$$
n-x=\sum_{i=1}^{k} \eta_{i} p^{i-1}
$$

$\left(\eta_{i} p^{i-1} \in D_{i}, i=1, \cdots, k\right)$, would belong to each set $E_{i}$, that is, $\eta_{i}=0$ for all $i$, which is a contradiction.

Finally, from the above construction,

$$
A_{i}(0, n) \geqq A_{i}\left(\frac{n}{2}, n-1\right) \geqq\left[\frac{n-1}{2}\right]-E_{i}\left(0, \frac{n-1}{2}\right) .
$$

But each $D_{h}$ has at most $p$ elements, thus, $E_{i}$ has at most $p^{k-1}$ elements; further, $0 \in E_{i}$, hence,

$$
k \frac{n-1}{2}-\sum_{i=1}^{k} A_{i}(0, n) \leqq k \frac{n-1}{2}-k\left[\frac{n+1}{2}\right]+k p^{k-1}<k p^{k-1} .
$$

In view of (1.4) and the definition of $p$, this proves (2.1).

Note that, for $n$ large, the above sets $A_{i}$ contain only very few elements smaller than $n / 2$. As will be seen, this is always the case for $k$-tuples satisfying (1.1), (1.3), which nearly attain the minimum in (1.4).

3. It remains to derive lower bounds on $\phi_{k}(n)$. Let $A_{1}, \cdots, A_{k}$ denote given sets of non-negative integers, each containing zero. Put

$$
C=A_{1}+\cdots+A_{k}
$$

and

$$
B_{i}=A_{1}+\cdots+A_{i-1}+A_{i+1}+\cdots+A_{k} \quad(i=1, \cdots, k) .
$$

By $0 \in A_{h}(h=1, \cdots, k)$, we have

$$
0 \in A_{j} \subset B_{i} \subset C
$$

if $j \neq i$.

Thus, $C$ can be written as the disjoint union

$$
C=D \cup \Delta \cup C^{*},
$$

where 


$$
\begin{aligned}
& D=A_{1} \cap A_{2} \cap \cdots \cap A_{k}, \\
& \Delta=\left(B_{1} \cup B_{2} \cup \cdots \cup B_{k}\right) \cap \bar{D},
\end{aligned}
$$

and

$$
C^{*}=C \cap \bar{B}_{1} \cap \bar{B}_{2} \cap \cdots \cap \bar{B}_{k}
$$

here the bar denotes complementation.

LEMMA 3.1. One has

$$
\Delta=\bigcup_{i=1}^{k}\left(B_{i} \cap A_{j_{i}}\right)
$$

for each permutation $\left(j_{1}, \cdots, j_{k}\right)$ of $(1, \cdots, k)$.

Proof. Let $f_{j}(x)$ denote the characteristic function of $A_{j}$, that is, $f_{j}(x)=1$ if $x \in A_{j}, f_{j}(x)=0$ otherwise. Similarly, let $g_{i}(x)$ denote the characteristic function of $\bar{B}_{i}$. By (3.3),

$$
g_{i}(x) f_{j}(x)=0
$$

if $i \neq j$;

thus,

$$
g_{i}(x) g_{j}(x) f_{h}(x)=0, \quad g_{h}(x) f_{i}(x) f_{j}(x)=0,
$$

whenever $\mathrm{i} \neq j$. Consequently, by $k \geqq 3$,

$$
\prod_{i=1}^{k}\left(g_{i}(x)+f_{j_{i}}(x)\right)=\prod_{i=1}^{k} g_{i}(x)+\prod_{j=1}^{k} f_{j}(x) \text {. }
$$

Given $x$, the latter right-hand side is equal to 0 if and only if $x \in \Delta$, by (3.5) and (3.6). The left-hand side is equal to 0 if and only if $x \in B_{i} \cap \bar{A}_{j_{t}}$ for some $i=1, \cdots, k$. This proves (3.8).

A perhaps more intuitive equivalent proof of Lemma 3.1 was suggested by the referee. Clearly, $\Delta$ is precisely the union of the $k^{2}$ sets $B_{h} \cap A_{j_{i}}$ and thus contains the right-hand side $\Delta^{1}$ (say) of (3.8). Conversely, let $x \in B_{h} \cap A_{j_{i}}$. In proving $x \in \Delta^{1}$, we may assume that

$$
x \notin B_{i}, \quad x \in A_{j_{h}} .
$$

It follows by (3.3) that $x \in B_{r}$ when $r \neq j_{h}$, thus, $i=j_{h}$, and further that $x \notin A_{j_{r}}$ when $j_{r} \neq i=j_{h}$, that is, when $r \neq h$. Therefore, $x \in B_{r} \cap A_{j_{r}}$ for each index $r$ with $r \neq i$ and $r \neq h$, in particular, $x \in \Delta^{1}$.

The following result is an immediate consequence of Theorem 5.1, which will be proved in $\S 5$. Here, and in the sequel, if $E$ is a set then $[E]$ denotes the number of elements in $E$.

LEMMA 3.2. Let $E$ be an arbitrary subset of $C$ and let 


$$
F_{i}=\left\{b_{i} \in B_{i}: a_{i}+b_{i} \in E \text { for some } a_{i} \in A_{i}\right\} \text {. }
$$

Then

$$
[E] \leqq\left(\left[F_{1}\right]\left[F_{2}\right] \cdots\left[F_{k}\right]\right)^{1 /(k-1)}
$$

This in turn implies

$$
[E]^{(k-1) / k} \leqq \frac{1}{k} \sum_{i=1}^{k}\left[F_{i}\right]
$$

Let $x$ denote a fixed positive integer, and let us apply Lemma 3.2 with

$$
E=\left\{c \in C^{*}: c \leqq x\right\}
$$

By (3.3) and (3.7), $0 \notin E$, hence

$$
[E]=C^{*}(0, x) \text {. }
$$

Consider a fixed element $c \in E \subset C^{*} \subset C$. By (3.2) and (3.7), if

$$
c=a_{1}+a_{2}+\cdots+a_{k}, \quad a_{i} \in A_{i},
$$

then necessarily $a_{i}>0(i=1, \cdots, k)$. Letting $c=a_{i}+b_{i}$, one has $b_{i} \in F_{i} \subset B_{i}$ and $0<b_{i} \leqq c-1 \leqq x-1$. Moreover, in each representation

$$
b_{i}=a_{1}^{\prime}+\cdots+a_{i-1}^{\prime}+a_{i+1}^{\prime}+\cdots+a_{k}^{\prime},
$$

(with $a_{j}^{\prime} \in A_{j}$ for $j \neq i$ ), one necessarily has $a_{j}^{\prime}>0$ for each $j \neq i$. In particular, by $k \geqq 3, b_{i} \notin A_{j}$ for each $j \neq i$. Hence, by (3.9),

$$
F_{i} \subset\left\{b_{i} \in B_{i}: 0<b_{i} \leqq x-1, b_{i} \notin A_{j} \text { for each } j \neq i\right\} .
$$

Thus, (3.10) yields

LEMMA 3.3. For each positive integer $x$,

$$
C^{*}(0, x)^{(k-1) / k} \leqq \frac{1}{k} \sum_{i=1}^{k}\left\{B_{i} \cap \bar{A}_{j \neq i}\right\}(0, x-1) .
$$

Let us now assume that

$$
n \notin C .
$$

This is equivalent to

$$
n \notin A_{i}+B_{i} \quad(i=1, \cdots, k) .
$$

Hence, by (3.3), $n \notin A_{i}$ and $n \notin B_{i}$, thus,

$$
A_{i}(0, n)=A_{i}(0, n-1), \quad B_{i}(0, n)=B_{i}(0, n-1) .
$$

By (3.13), if $x<y$ are real numbers and $b \in B_{i}, x<b \leqq y$ then $n-b \notin A_{i}$, $n-[y] \leqq n-b<n-[x]$. Therefore, 


$$
B_{i}(x, y) \leqq[y]-[x]-A_{i}(n-1-[y], n-1-[x]) .
$$

In particular,

$$
B_{i}(0, n-1) \leqq n-1-A_{i}(0, n-1) .
$$

Moreover, by (3.3), $A_{i+1} \subset B_{i}\left(A_{k+1}=A_{1}\right)$; consequently,

$$
\sum_{i=1}^{k}\left\{B_{i} \cap \bar{A}_{i+1}\right\}(0, n) \leqq 2 \rho
$$

(thus, $\rho \geqq 0$ ), where $\rho$ is defined by

$$
\sum_{i=1}^{k} A_{i}(0, n)=k \frac{n-1}{2}-\rho .
$$

By (3.8) and (3.15),

$$
\Delta(0, n) \leqq 2 \rho .
$$

By (3.11) and (3.15),

$$
C^{*}(0, n) \leqq(2 \rho / k)^{k /(k-1)} .
$$

Finally, by (3.5) and (3.16),

Thus, by (3.4):

$$
D(0, n) \leqq \min _{i} A_{i}(0, n) \leqq \frac{n-1}{2}-\frac{\rho}{k} .
$$

THEOREM 3.4. Suppose that (3.12) and (3.16) hold. Then

$$
C(0, n) \leqq \frac{n-1}{2}+\left(2-\frac{1}{k}\right) \rho+(2 \rho / k)^{k /(k-1)} .
$$

If $\rho=0$ then $C(0, n) \leqq(n-1) / 2$. But $A_{i} \subset C$; thus, by (3.16), $\rho=0$ implies that below $n$ the sets $A_{i}$ and $C$ constitute one and the same set $A$, say, consisting of 0 and further $(n-1) / 2$ positive integers $<n$. Moreover, $a, a^{\prime} \in A$ implies that either $a+a^{\prime} \in A$ or $a+a^{\prime}>n$. Conversely, if $n$ is odd and $A$ is any such set then the choice $A_{i}=A(i=1, \cdots, k)$ yields a $k$-tuple satisfying $n \notin C$ and $\rho=0$. Typical examples are

and

$$
A=\{0, m+1, m+2, \cdots, 2 m\}
$$

$$
A=\{0,2,4, \cdots, 2 m\},
$$

where $m=(n-1) / 2$. In general, $n=2 m+1 \notin A$ implies that either $m \notin A$ or $m+1 \notin A$ which in turn implies

$$
A\left(0, \frac{n-1}{2}\right) \leqq \frac{n-1}{4} \quad(\text { when } \rho=0) .
$$


If (besides (3.12)) we assume that

$$
\{0,1,2, \cdots, n-1\} \subset C
$$

then $C(0, n)=n-1$. In the more general case where we assume that

(3.18) yields

$$
C(0, n) \geqq\left(\frac{1}{2}+\frac{1}{p}\right)(n-1) \quad(p \geqq 2),
$$

\section{(3.18) yields}

$$
\left(\frac{2 \rho}{k}\right)^{k /(k-1)} \geqq \frac{n-1}{p}-\frac{2 k-1}{2}\left(\frac{n-1}{p}\right)^{(k-1) / k} .
$$

After all, if (3.21) were false then

$$
\left(2-\frac{1}{k}\right) \rho=\frac{2 k-1}{2} \frac{2 \rho}{k}<\frac{2 k-1}{2}\left(\frac{n-1}{p}\right)^{(k-1) / k} \text {. }
$$

It follows from (3.21) (with $p=2),(3.16)$ and (1.4) that

$$
\phi_{k}(n) \geqq \frac{1}{2} k\left(\frac{n-1}{2}\right)^{(k-1) / k}-O\left(n^{(k-2) / k}\right) .
$$

Together with (2.1) this yields for $n$ large an estimate of the type (1.5) with

$$
\beta_{k}=k\left(\frac{1}{2}\right)^{(k-1) / k}, \quad \alpha_{k}=\frac{1}{2} \beta_{k} \text {. }
$$

4. In this section, the lower bound (3.22) will be improved so as to demonstrate Theorem 1.1. As a byproduct, we obtain some additional information on the structure of the "optimal" $k$-tuples.

Let again $A_{1}, \cdots, A_{k}$ be sets of non-negative integers such that $0 \in A_{i}$ and

$$
n \notin C=A_{1}+\cdots+A_{k} .
$$

Further, $\rho \geqq 0$ will always be defined by

$$
\sum_{i=1}^{n} A_{i}(0, n)=k \frac{n-1}{2}-\rho .
$$

Applying (3.14) with $x=0, y=(n-1) / 2$, we have

$$
B_{i}\left(0, \frac{n-1}{2}\right) \leqq\left[\frac{n-1}{2}\right]-A_{i}\left(\left[\frac{n}{2}\right], n-1\right) .
$$

By $A_{i+1} \subset B_{i},\left(A_{k+1}=A_{1}\right)$, this yields

$$
\sum_{i=1}^{k}\left\{B_{i} \cap A_{i+1}\right\}\left(0, \frac{n-1}{2}\right) \leqq k\left[\frac{n-1}{2}\right]-\sum_{i=1}^{k}\left(A_{i}(0, n-1)-\varepsilon_{i}\right),
$$

where $\varepsilon_{i}=1$ if $n / 2 \in A_{i}, \varepsilon_{i}=0$ otherwise. But $\varepsilon_{i}=1$ can only happen when $n$ is even and then only for at most one index $i$, by $n \notin C$. Therefore, using (4.2), 


$$
\sum_{i=1}^{k}\left\{B_{i} \cap \bar{A}_{i+1}\right\}\left(0, \frac{n-1}{2}\right) \leqq \rho .
$$

It follows by (3.11) that

$$
C^{*}\left(0, \frac{n+1}{2}\right) \leqq(\rho / k)^{k /(k-1)}
$$

and by (3.8) that

$$
\Delta\left(0, \frac{n-1}{2}\right) \leqq \rho .
$$

Remember (cf. (3.17)) that

$$
\Delta(0, n-1) \leqq 2 \rho .
$$

Definition. With $q$ as a positive integer, let $S_{q}$ denote the set of all integers $1 \leqq m \leqq(n-1) / 2$ admitting at least one representation of the form

$$
m=a_{1}+a_{2}+\cdots+a_{q} \text { with } a_{j} \in \bigcup_{i=1}^{k} A_{i}(j=1, \cdots, q) .
$$

Let further $\lambda_{q} \geqq 0$ denote the number of integers $1 \leqq m \leqq(n-1) / 2$ with $m \notin S_{q}$. Note that, by $0 \in A_{i}$, we have $S_{q+1} \supset S_{q}$ and $\lambda_{q+1} \leqq \lambda_{q}$.

THEOREM 4.1. We have for each positive integer $q$ that

$$
D\left(0, \frac{n-1}{2}\right) \leqq\left(1+\frac{1}{\sqrt{ } 2}\right) \lambda_{q}+(4+2 \sqrt{ } 2) q \rho .
$$

Let us first establish a few lemmas.

LEMMA 4.2. Let $m \in S_{q}$. Then

(i) There are at most $2 q \rho$ elements $d \in D$ such that $0 \leqq d \leqq(n-1) / 2$ and $d+m \notin D$.

(ii) There are at most $q \rho$ elements $d \in D$ such that $0 \leqq d \leqq(n-1) / 2-m$ and $d+m \notin D$.

Proof. Let (4.8) be a fixed representation of $m \in S_{q}$. Consider an element $d \in D$ such that $0 \leqq d \leqq(n-1) / 2$ and $d+m \notin D$. There exists a smallest index $1 \leqq r \leqq q$ such that

$$
d^{\prime}=d+a_{1}+\cdots+a_{r-1} \in D \text { and } d^{\prime}+a_{r} \notin D .
$$

If $a_{r} \in A_{j}$ and $h \neq j$ then $d^{\prime}+a_{r} \in B_{h}$ (choose $1 \leqq i \leqq k$ distinct from $j$ and $h$ and observe that $d^{\prime} \in D \subset A_{i}$ ). Hence, by (3.6), $d^{\prime}+a_{r} \in \Delta$. Moreover, $d^{\prime}+a_{r} \leqq d+m \leqq n-1$; thus, by (4.7), we have for each fixed $1 \leqq r \leqq q$ that 
(4.9) can happen for at most $2 \rho$ elements $d$. This proves assertion (i). In a similar way, (4.6) yields assertion (ii).

Lemma 4.3. Let $m \in S_{q}$. Then

$$
D\left(\frac{n-1}{2}-m, \frac{n-1}{2}\right) \leqq \frac{m}{2}+q \rho .
$$

Hence,

$$
D\left(0, \frac{n-1}{2}-m\right) \geqq N-\frac{m}{2}-q \rho,
$$

where

$$
N=D\left(0, \frac{n-1}{2}\right)
$$

Proof. Consider the sets

$$
D^{\prime}=\left\{d \in D:\left[\frac{n+1}{2}\right]-m \leqq d \leqq\left[\frac{n}{2}\right]\right\}
$$

and

$$
D^{\prime \prime}=\left\{d \in D:\left[\frac{n+1}{2}\right] \leqq d \leqq\left[\frac{n}{2}\right]+m\right\}
$$

Notice that

$$
\left[\frac{n+1}{2}\right]+\left[\frac{n}{2}\right]=n
$$

Hence, by $n \notin C, D+D \subset C$, one has

$$
\left[D^{\prime}\right]+\left[D^{\prime \prime}\right] \leqq m
$$

(if $n$ is even, then $n / 2 \notin D$ ).

On the other hand, by Lemma 4.2, one has for all but $2 q \rho$ elements $d \in D^{\prime}$ that $d+m \in D^{\prime \prime}$, thus, $\left[D^{\prime \prime}\right] \geqq\left[D^{\prime}\right]-2 q \rho$; therefore,

$$
m \geqq\left[D^{\prime}\right]+\left[D^{\prime}\right]-2 q \rho,
$$

implying (4.10).

LEMMA 4.4. If $m \in S_{q}$ then there are at least

$$
N+1-\frac{m}{2}-2 q \rho
$$

pairs of elements $d, d^{\prime}$ in $D$ such that

$$
0 \leqq d<d^{\prime} \leqq \frac{n-1}{2}, \quad d^{\prime}-d=m .
$$


Proof. By (4.11) and $0 \in D$, there are at least $N+1-m / 2-q \rho$ elements $d \in D$ with $0 \leqq d \leqq(n-1) / 2-m$. By Lemma 4.2 , we have for all but at most $q \rho$ of these elements that $d^{\prime}=d+m \in D$.

The following generalizes (3.19).

LEMMA 4.5. One has

$$
N \leqq \frac{n-1}{4}+2 \rho
$$

Proof. Let $d_{0}$ denote the largest element $d_{0} \in D$ with $0<d_{0} \leqq(n-1) / 2$ (if no such elements exist then $N=0$ ). Notice that (4.13) with $m=d_{0}, d^{\prime} \in D$ is only possible with $d=0, d^{\prime}=d_{0}$. Hence, applying Lemma 4.4 with $m=d_{0} \in S_{1}$, one obtains that

$$
N+1-\frac{d_{0}}{2}-2 \rho \leqq 1
$$

proving (4.14).

Proof of Theorem 4.1. There are precisely $N+1$ elements $d \in D$ with $0 \leqq d \leqq(n-1) / 2$, hence, precisely $\frac{1}{2} N(N+1)$ pairs $d, d^{\prime}$ in $D$ with $0 \leqq d<d^{\prime} \leqq(n-1) / 2$. It follows by Lemma 4.4 that

$$
\text { (4.15) } \frac{1}{2} N(N+1) \geqq \sum_{m \in T}\left(N+1-\frac{m}{2}-2 q \rho\right)=[T](N+1-2 q \rho)-\sum_{m \in T} \frac{m}{2}
$$

is true for any subset $T$ of $S_{q}$. We shall choose

$$
T=\left\{m \in S_{q}: 1 \leqq m \leqq r\right\},
$$

where $r$ denotes the integer defined by

$$
r=2 N-4 q \rho \text {. }
$$

One may assume that $r>0$; otherwise, (4.9) is obvious. Further, $r \leqq(n-1) / 2$ by (4.14) and $q \geqq 1$. It follows from the definition of $\lambda_{q}$ that

$$
[T]=r-\lambda_{q}^{\prime} \text { with } 0 \leqq \lambda_{q}^{\prime} \leqq \lambda_{q} \text {. }
$$

Moreover, by (4.15),

$$
\frac{1}{2} N(N+1) \geqq\left(r-\lambda_{q}^{\prime}\right)(N+1-2 q \rho)-\sum_{m=1}^{r} \frac{m}{2}+\sum_{m=1}^{\lambda_{q}^{\prime}} \frac{m}{2} .
$$

Substituting (4.16), one obtains the inequality

$$
\frac{1}{2} N^{2}-\alpha N+\beta \leqq 0,
$$

where 


$$
\alpha=\gamma-1, \quad \beta=\frac{1}{4} \gamma(\gamma-3) .
$$

Here,

$$
\gamma=4 q \rho+\lambda_{q}^{\prime}, \text { thus, } 0 \leqq \gamma \leqq 4 q \rho+\lambda_{q}
$$

Note that

$$
0 \leqq \alpha^{2}-2 \beta=\frac{1}{2} \gamma^{2}-\frac{1}{2} \gamma+1 \leqq\left(\frac{1}{\sqrt{ } 2} \gamma+1\right)^{2} .
$$

Consequently, by (4.17),

$$
N \leqq \alpha+\sqrt{ }\left(\alpha^{2}-2 \beta\right) \leqq(\gamma-1)+\left(\frac{1}{\sqrt{ } 2} \gamma+1\right) \leqq\left(1+\frac{1}{\sqrt{ } 2}\right)\left(4 q \rho+\lambda_{q}\right) .
$$

This proves Theorem 4.1.

Theorem 1.1 is an easy consequence of Theorem 4.1. As before, suppose $n \notin C$ and let $\rho$ be defined by (4.2). Instead of (1.3), let us merely assume that

or still less, that

$$
\left\{0,1, \cdots,\left[\frac{n-1}{2}\right]\right\} \subset C
$$

$$
C\left(0, \frac{n-1}{2}\right) \geqq\left[\frac{n-1}{2}\right]-\lambda(n),
$$

where $\{\lambda(n)\}$ is a given sequence of positive numbers such that

$$
\lim _{n \rightarrow \infty} \frac{\lambda(n)}{n}=0
$$

Noting that $C \subset S_{k}$, we have that (4.18) implies $\lambda_{k} \leqq \lambda(n)$. Hence, by (3.4), (4.5), (4.6) and (4.9) (with $q=k$ ),

$$
\left[\frac{n-1}{2}\right]-\lambda(n) \leqq\left(1+\frac{1}{\sqrt{ } 2}\right) \lambda(n)+(4+2 \sqrt{ } 2) k \rho+\rho+(\rho / k)^{k /(k-1)} .
$$

It follows that

$$
(\rho / k)^{k /(k-1)} \geqq \frac{n}{2}-o(n)
$$

Thus,

$$
\lim _{n \rightarrow \infty} \inf ^{-(k-1) / k} \min \rho \geqq k\left(\frac{1}{2}\right)^{(k-1) / k}
$$

(here, for each fixed $n$, the minimum is subject to $n \notin C$ and (4.18)). In view of (1.4), (2.1) and (4.2), this proves Theorem 1.1.

Notice that a $k$-tuple $\left\{A_{1}, \cdots, A_{k}\right\}$ which satisfies $n \notin C$ and (4.18), and which is nearly optimal in the sense that 


$$
\rho=o(n),
$$

satisfies $D(0,(n-1) / 2)=o(n)$, by (4.9) with $q=k$; hence, $A_{i}(0,(n-1) / 2)=o(n)$, by (3.3), (3.6) and (4.6); hence,

$$
A_{i}\left(\frac{n-1}{2}, n-1\right)=\frac{n-1}{2}-o(n) \quad(i=1, \cdots, k)
$$

therefore, $C(0, n-1)=n-1-o(n)$. In other words, for optimal $k$-tuples, the assumption (4.18) nearly implies the much stronger (1.3).

If even $(\rho / k)^{k /(k-1)} \sim n / 2$ then (4.5) holds nearly with the equality sign, suggesting that the part of $C^{*}$ below $n / 2$ does not deviate much from a direct sum with nearly disjoint components of about equal size, compare Theorem 5.1 and the way it was used in the proof of (4.5).

The reasoning leading to (4.20) can easily be adapted in proving the following result.

Let $\{\lambda(n)\}$ be as in (4.19) and let $\{q(n)\}$ be a sequence of positive integers satisfying $q(n) \rightarrow \infty, q(n)=o\left(n^{1 / k}\right)$. Let further $0<\varepsilon \leqq 1$ be a given positive constant and let

$$
\psi_{k}(n, \varepsilon)=\min \left\{k \frac{n-1}{2}-\sum_{i=1}^{n} A_{i}(0, n)\right\},
$$

where $\left\{A_{1}, \cdots, A_{k}\right\}$ ranges over the $k$-tuples of sets $A_{i}$ of non-negative integers such that $0 \in A_{i}, n \notin C$,

$$
C\left(0, \frac{n-1}{2}\right) \geqq \varepsilon\left[\frac{n-1}{2}\right]
$$

and

$$
\lambda_{q(n)} \leqq \lambda(n)
$$

THEOREM 4.6. With the above notations,

$$
\lim _{n \rightarrow \infty} \psi_{k}(n, \varepsilon) n^{-(k-1) / k}=k(\varepsilon / 2)^{(k-1) / k} .
$$

Here, an upper bound on $\rho$ is easily obtained from the construction in $\S 2$, by replacing there $p$ by the smallest integer satisfying

$$
\varepsilon \frac{n}{2} \leqq p^{k}-1
$$

(the resulting $k$-tuple has $\lambda_{k h}=0$ as soon as $\left.h[\varepsilon n / 2] \geqq(n-1) / 2\right)$.

The restriction (4.23) cannot be omitted. For, take each $A_{i}$ as the set $A$ obtained from $\{[(n+1) / 2], \cdots, n-1\}$ by adding a block of multiples $r t$ of $t$ immediately 
below $[(n+1) / 2]$ and deleting the corresponding elements $n-r t$; here, $t$ is a positive integer not dividing $n$. In this example, $\lambda_{q}$ is independent of $q$.

5. Let $G$ be an arbitrary commutative semigroup, written additively (that is, a set together with an associative and commutative addition). Lemma 3.2 is an immediate consequence of:

THEOREM 5.1. Be given $k \geqq 2$ finite and nonempty subsets $A_{1}, \cdots, A_{k}$ of $G$. Put

$$
C=A_{1}+\cdots+A_{k}, \quad B_{i}=A_{1}+\cdots+A_{i-1}+A_{i+1}+\cdots+A_{k}
$$

$(i=1, \cdots, k)$. Let $E$ be any subset of $C$ and put

$$
B_{i}^{*}=\left\{b_{i} \in B_{i}: a_{i}+b_{i} \in E \text { for some } a_{i} \in A_{i}\right\}
$$

Then

$$
[E] \leqq\left(\left[B_{1}^{*}\right]\left[B_{2}^{*}\right] \cdots\left[B_{k}^{*}\right]\right)^{1 /(k-1)} .
$$

Here, the equality sign holds if and only if $E$ is the direct sum of the $k$ sets

$$
A_{i}^{*}=\left\{a_{i} \in A_{i}: a_{i}+b_{i} \in E \text { for some } b_{i} \in B_{i}\right\}
$$

More precisely, $E$ consists of all elements $c$ of the form $c=a_{1}^{*}+\cdots+a_{k}^{*}\left(a_{i}^{*} \in A_{i}^{*}\right)$; moreover, given $c \in E$, this representation is unique. In particular, $[E]=\left[A_{1}^{*}\right] \cdots\left[A_{k}^{*}\right]$; if a point $a_{0}$ is in two $A_{i}^{*}$ it is in all $A_{i}^{*}$, and there can be only one such $a_{0}$.

For the proof of Theorem 5.1 we shall need the following lemma, which is contained in the more general Theorem 5.3. In the sequel, $k$ denotes an integer, $k \geqq 2$.

LEMMA 5.2. In the $k$-dimensional Euclidean space $\boldsymbol{R}^{k}$, be given a rectangular coordinate system. Let $D$ be any finite subset of $R^{k}$ and let $D_{1}, \cdots, D_{k}$ denote the projections of $D$ on the $(k-1)$-dimensional coordinate planes. Then

$$
[D] \leqq \sqrt[k-1]{\left[D_{1}\right]\left[D_{2}\right] \cdots\left[D_{k}\right]}
$$

Here, the equality sign holds if and only if $D$ coincides with the direct product of its projections on the $k$ coordinate axes.

Proof of Theorem 5.1. Let the elements of $A_{i}$ be enumerated in some arbitrary but fixed fashion

$$
A_{i}=\left\{a_{i}(1), a_{i}(2), \cdots, a_{i}\left(\left[A_{i}\right]\right)\right\}
$$

$(i=1, \cdots, k)$. Consider an element $c \in E$. It admits one or more representations of the form

$$
c=a_{1}\left(j_{1}\right)+a_{2}\left(j_{2}\right)+\cdots+a_{k}\left(j_{k}\right) .
$$


Choose $j_{1}$ as small as possible. If $j_{1}, \cdots, j_{i-1}$ have been chosen already, choose $j_{i}$ as small as possible, $i=1, \cdots, k$ (if the cancellation law does not hold, $j_{k}$ is not yet determined by the other $j_{i}$ ). The resulting unique representation (5.3) of $c$ will be called the canonical representation of $c$ (cf. [1, p. 51]).

When (5.3) is the canonical representation of $c \in E$, put

$$
\hat{c}=\left(j_{1}, \cdots, j_{k}\right) \text {. }
$$

In this way there corresponds to $E$ a set $D$ of lattice points $\hat{c}$ in $R^{k},[D]=[E]$. By Lemma 5.2, it suffices to prove that

$$
\left[D_{i}\right] \leqq\left[B_{i}^{*}\right] \quad(i=1, \cdots, k),
$$

where $D_{1}, \cdots, D_{k}$ denote the projections of $D$ on the $(k-1)$-dimensional coordinate planes. That is, $D_{i}$ is the set of all points

$$
d_{i}=\left(j_{1}, j_{2}, \cdots, j_{i-1}, 0, j_{i+1}, \cdots, j_{k}\right)
$$

with integer coordinates, $1 \leqq j_{s} \leqq\left[A_{s}\right]$ if $s \neq i$, such that, for at least one choice of $j_{i},(5.3)$, is the canonical representation of some element $c \in E$.

In proving (5.3), it suffices to construct a $1: 1$ mapping of $D_{i}$ into $B_{i}^{*}$ ( $i$ fixed). Namely, if $d_{i} \in D_{i}$ is given by (5.4), let

$$
\phi\left(d_{i}\right)=a_{1}\left(j_{1}\right)+\cdots+a_{i-1}\left(j_{i-1}\right)+a_{i+1}\left(j_{i+1}\right)+\cdots+a_{k}\left(j_{k}\right) .
$$

Clearly, by (5.1) and the above property of $d_{i}$, one has $\phi\left(d_{i}\right) \in B_{i}^{*}$. Let

$$
d_{i}^{\prime}=\left(j_{1}^{\prime}, \cdots, j_{i-1}^{\prime}, 0, j_{i+1}^{\prime}, \cdots, j_{k}^{\prime}\right)
$$

be a further point in $D_{i}$, such that $\phi\left(d_{i}\right)=\phi\left(d_{i}^{\prime}\right)$. We must prove that $d_{i}=d_{i}^{\prime}$. Let $1 \leqq s \leqq k, s \neq i$, be such that $j_{\lambda}^{\prime}=j_{\lambda}$ for $\lambda<s, \lambda \neq i$.

By $d_{i} \in D_{i}$, there exists an integer $j_{i}$ such that (5.3) is the canonical representation of an element $c \in E$. By $\phi\left(d_{i}\right)=\phi\left(d_{i}^{\prime}\right)$,

$$
c=a_{i}\left(j_{i}\right)+\phi\left(d_{i}^{\prime}\right)=a_{1}\left(j_{1}^{\prime}\right)+\cdots+a_{k}\left(j_{k}^{\prime}\right),
$$

where $j_{i}^{\prime}=j_{i}$; thus, $j_{\lambda}^{\prime}=j_{\lambda}$ for $\lambda<s$. It follows from the minimal character of $j_{s}$ that $j_{s}^{\prime} \geqq j_{s}$. Similarly, $j_{s} \geqq j_{s}^{\prime}$; hence, $j_{s}^{\prime}=j_{s}$. It follows that $j_{\lambda}^{\prime}=j_{\lambda}$ for all $\lambda \neq i$; thus, $d_{i}^{\prime}=d_{i}$.

If (5.2) holds with the equality sign then, by the last assertion of Lemma 5.2, $D$ is the direct product of its projections in the coordinate axes; moreover, $\left[D_{i}\right]=\left[B_{i}^{*}\right]$ $(i=1, \cdots, k)$. This easily implies the last assertion of Theorem 5.1.

TheOrem 5.3. Let $D$ be a given Lebesgue measurable subset of $R^{k}$. Let $D_{1}, \cdots, D_{k}$ denote the essential projections of $D$ on the $(k-1)$-dimensional coordinate planes of a given rectangular coordinate system. Then

$$
\mu_{k}(D) \leqq\left[\prod_{i=1}^{k} \mu_{k-1}\left(D_{i}\right)\right]^{1 /(k-1)}
$$


Here, the equality sign holds if and only if $D$ differs by at most a set of $\mu_{k}$ measure 0 from a direct product $A_{1} \times \cdots \times A_{k}\left(A_{i}\right.$ denoting a measurable set on the ith coordinate axis).

Here, $\mu_{r}$ denotes $r$-dimensional Lebesgue measure. By the essential projection $D_{i}$ of $D$ we mean the set of points $d_{i}=\left(x_{1}, \cdots, x_{i-1}, 0, x_{i+1}, \cdots, x_{k}\right)$ such that the line through $d_{i}$ parallel to the $i$ th coordinate axis intersects $D$ in a set of positive linear outer measure. By Fubini, $D_{i}$ is a Lebesgue measurable set. If

$$
\begin{aligned}
f(x)=f\left(x_{1}, \cdots, x_{n}\right) & =1 & & \text { if } x \in D, \\
& =0 & & \text { if } x \notin D,
\end{aligned}
$$

denotes the characteristic function of $D$ then the (measurable) function

$$
g_{i}(x)=g_{i}\left(x_{1}, \cdots, x_{i-1}, x_{i+1}, \cdots, x_{k}\right)=\text { ess sup } \operatorname{xup}_{i} f\left(x_{1}, \cdots, x_{k}\right)
$$

is precisely the characteristic function of $D_{i}, i=1, \cdots, k$; (deleting from $D$ a suitable set of measure zero, one may replace in (5.7) the essential supremum by the ordinary supremum).

Lemma 5.2 follows from Theorem 5.3 by describing about each point, of the finite set $D$ in $R^{k}$, a small cube of fixed size having its edges parallel to the coordinate axes.

As far as I am aware, the isoperimetric inequality (5.5) is new. A related result due to Ohmann [2] gives as the upper bound on $\mu_{k}(D)^{1-1 / k}$ the integral over all directions $w$ of the $(k-1)$-dimensional Lebesgue measure of the $w$-projection of $D$, multiplied by a constant such the resulting upper bound is attained for a solid sphere.

Proof of Theorem 5.3. We may assume that $\mu_{k}(D)>0$. The proof goes by induction with respect to $k$. We may assume that $k \geqq 3$, (the assertion being obvious for $k=2$ ), and further that the assertion is true when $k$ is replaced by $k-1$. Finally (applying a suitable transformation $x_{i}^{\prime}=c_{i} x_{i}, i=1, \cdots, k$, to $D$ ), one may assume that

$$
\int g_{i}(x) d x_{1} \cdots d x_{i-1} d x_{i+1} \cdots d x_{k}=\mu_{k-1}\left(D_{i}\right)=1
$$

$(i=1, \cdots, k$, in particular for $i=k)$. Thus, in view of (5.7),

$$
\int f\left(x_{1}, \cdots, x_{k}\right) d x_{1} d x_{2} \cdots d x_{k-1} \leqq 1,
$$

for almost all $x_{k}$. Moreover, by (5.7) and induction,

$$
\int f\left(x_{1}, \cdots, x_{k}\right) d x_{1} \cdots d x_{k-1} \leqq\left[\prod_{i=1}^{k-1} h_{i}\left(x_{k}\right)\right]^{1 /(k-2)},
$$

for almost all $x_{k}$, where 


$$
h_{i}\left(x_{k}\right)=\int g_{i}(x) d x_{1} \cdots d x_{i-1} d x_{i+1} \cdots d x_{k-1} \quad(i=1, \cdots, k-1) .
$$

Note that, by (5.8),

$$
\int h_{i}\left(x_{k}\right) d x_{k}=1 \quad(i=1, \cdots, k-1)
$$

As is well known, $u_{i} \geqq 0(i=1, \cdots, k-1)$ implies

$$
\left(u_{1} u_{2} \cdots u_{k-1}\right)^{1 /(k-1)}<\left(u_{1}+\cdots+u_{k-1}\right) /(k-1)=c,
$$

say, unless the $u_{i}$ are all equal to each other. Distinguishing between $c>1$ and $c \leqq 1$, it follows that

$$
\min \left(1,\left(u_{1} \cdots u_{k-1}\right)^{1 /(k-2)}\right) \leqq\left(u_{1}+\cdots+u_{k-1}\right) /(k-1),
$$

the equality sign holding if and only if either $u_{i}=0$ for all $i$ or $u_{i}=1$ for all $i$. Replacing $u_{i}$ by $h_{i}\left(x_{k}\right), i=1, \cdots, k-1$, it follows by (5.9) and (5.10) that

$$
\int f\left(x_{1}, \cdots, x_{k}\right) d x_{1} \cdots d x_{k-1} \leqq\left(h_{1}\left(x_{k}\right)+\cdots+h_{k-1}\left(x_{k}\right)\right) /(k-1),
$$

for almost all $x_{k}$. Integrating with respect to $x_{k}$ and using (5.11), one obtains that $\mu_{k}(D) \leqq 1$.

Suppose that $\mu_{k}(D)=1$. Then for almost all $x_{k}$ we have the following. First, either $h_{i}\left(x_{k}\right)=0$ for all $i$ (thus, the left-hand side of (5.12) is equal to zero), or $h_{i}\left(x_{k}\right)=1$ for all $i$ and the left-hand side of (5.12) is equal to 1 , thus, by (5.7) and (5.8),

$$
f\left(x_{1}, \cdots, x_{k-1}, x_{k}\right)=g_{k}\left(x_{1}, \cdots, x_{k-1}\right),
$$

for almost all $\left(x_{1}, \cdots, x_{k-1}\right)$. Moreover (in the latter case), (5.10) holds with the equality sign; hence, by induction,

$$
g_{k}\left(x_{1}, \cdots, x_{k-1}\right)=\chi_{A_{1}}\left(x_{1}\right) \cdots \chi_{A_{k-1}}\left(x_{k-1}\right),
$$

for almost all $\left(x_{1}, \cdots, x_{k-1}\right)$. Here, $A_{i}$ denotes a linear set with characteristic function $\chi_{A_{i}}(x), i=1, \cdots, k-1$. Letting $A_{k}$ denote the set of those numbers $x_{k}$ for which the left-hand side of (5.12) is equal to 1 , it follows that

$$
f(x)=f\left(x_{1}, \cdots, x_{k}\right)=\chi_{A_{1}}\left(x_{1}\right) \cdots \chi_{A_{k-1}}\left(x_{k-1}\right) \chi_{A_{k}}\left(x_{k}\right),
$$

for almost all $x=\left(x_{1}, \cdots, x_{k}\right)$.

By a different, somewhat less intuitive proof, the inequality (5.5) can be generalized as follows. For $i=1, \cdots, k$, let

$$
\left(\Omega_{i}, \mathscr{B}_{i}, v_{i}\right)
$$

be a $\sigma$-finite measure space ( $v_{i}$ a non-negative measure on the $\sigma$-field $\mathscr{B}_{i}$; we shall assume that $\mathscr{B}_{i}$ is complete relative to $v_{i}$ ). Let their direct product be denoted as 


$$
\left(\Omega, \mathscr{B}, v_{1} \times \cdots \times v_{k}=v\right),
$$

and let $\mathscr{B}^{\prime}$ denote the completion of $\mathscr{B}$ relative to $v$.

In the following, $x_{i}$ runs through $\Omega_{i}$; further, $d x_{i}$ denotes the integration $v_{i}\left(d x_{i}\right)$ relative to $x_{i}$ and $v_{i}, i=1, \cdots, k$.

Be given $k$ non-negative functions

$$
f_{i}(x)=f_{i}\left(x_{1}, \cdots, x_{k}\right) \geqq 0,
$$$$
i=1, \cdots, k \text {, }
$$

on $\Omega$, each measurable relative to $\mathscr{B}^{\prime}$. Define further

$$
M=\int \prod_{i=1}^{k} f_{i}(x) d x_{1} \cdots d x_{k}
$$

and

$$
M_{i}=\int\left[\operatorname{ess} \sup _{x_{i}} \int f_{i}(x)^{k-1} d x_{1} \cdots d x_{i-1}\right] d x_{i+1} \cdots d x_{k}
$$

and

$$
N=M^{1 / k}, \quad N_{i}=M_{i}^{1 /(k-1)}, \quad i=1, \cdots, k .
$$

THEOREM 5.4. One has

$$
M \leqq\left(M_{1} M_{2} \cdots M_{k}\right)^{1 /(k-1)}
$$

equivalently,

$$
N \leqq\left(N_{1} N_{2} \cdots N_{k}\right)^{1 / k}
$$

The discussion of the equality sign is more complicated in the present case and will be omitted. Taking each function $f_{i}$ equal to the characteristic function (5.6), Theorem 5.4 yields an inequality which is stronger than (5.5).

In proving Theorem 5.4, let us first consider the case $k=2$. One has

$$
\begin{aligned}
\int f_{1}\left(x_{1}, x_{2}\right) f_{2}\left(x_{1}, x_{2}\right) d x_{1} & \leqq\left[\operatorname{ess} \sup _{x_{1}} f_{1}\left(x_{1}, x_{2}\right)\right] \int f_{2}\left(x_{1}, x_{2}\right) d x_{1} \\
& \leqq\left[\operatorname{ess} \sup _{x_{1}} f_{1}\left(x_{1}, x_{2}\right)\right] \text { ess sup } \sin _{x_{2}} \int f_{2}\left(x_{1}, x_{2}\right) d x_{1},
\end{aligned}
$$

the latter inequality holding at least for almost $\left[v_{2}\right]$ values $x_{2}$. Integrating with respect to $x_{2}$ one obtains (5.17).

Next, let $k \geqq 3$ be given, and suppose that Theorem 5.4 holds when $k$ is replaced by $k-1$. Put

Letting

$$
I\left(x_{k}\right)=\int \prod_{i=1}^{k} f_{i}(x) d x_{1} \cdots d x_{k-1}
$$

$$
r=(k-1) /(k-2), s=k-1, \text { thus, } \frac{1}{r}+\frac{1}{s}=1 \text {, }
$$


it follows by Hölder's inequality that

$$
I\left(x_{k}\right) \leqq\left[\int\left\{\prod_{i=1}^{k-1} f_{i}(x)\right\}^{r} d x_{1} \cdots d x_{k-1}\right]^{1 / r} M_{k}^{1 /(k-1)},
$$

for almost $\left[v_{k}\right]$ all $x_{k}$; here, we also used (5.15) with $i=k$.

Applying (5.17) with $k$ replaced by $k-1$ to the first integral and noting that $(k-2) r=k-1$, one obtains that

$$
I\left(x_{k}\right) \leqq N_{k} \prod_{i=1}^{k-1} h_{i}\left(x_{k}\right)^{1 /(k-1)}
$$

for almost $\left[v_{k}\right]$ all $x_{k}$, where

$$
h_{i}\left(x_{k}\right)=\int\left[\underset{x_{i}}{\operatorname{ess} \sup _{i}} \int f_{i}(x)^{k-1} d x_{1} \cdots d x_{i-1}\right] d x_{i+1} \cdots d x_{k-1}
$$

$(i=1, \cdots, k-1)$. By (5.15) and (5.16),

$$
\left\{\int h_{i}\left(x_{k}\right) d x_{k}\right\}^{1 /(k-1)}=M_{i}^{1 /(k-1)}=N_{i} \quad(i=1, \cdots, k-1) .
$$

Hence, by (5.14), (5.19) and Hölder's inequality,

$$
N^{k}=M=\int I\left(x_{k}\right) d x_{k} \leqq N_{k}\left(N_{1} N_{2} \cdots N_{k-1}\right) .
$$

\section{REFERENCES} 45-55.

1. P. Erdös and P. Scherk, On a question of additive number theory, Acta Arith. 5 (1958),

2. D. Ohmann, Ungleichungen zwischen den Quermaszintegralen beschränkter Punktmengen, Math. Ann. 124 (1952), 265-276; ibid. 127 (1954), 1-7.

\section{UNIVERSITY OF ROCHESTER,}

ROCHESTER, NEW YORK 